\title{
COOLING FOR THE ARMATURE WINDINGS IN A REPETITIVELY
} FIRED AIR-CORE COMPULSATOR

\author{
H. -P. LiU
}

Presented at the 4th Symposium on Electromagnetic Launch Technology

Austin, Texas

Apri1 12-14, 1988

Publication No. PR-70

Center for Electromechanics

The University of Texas at Austin

Balcones Research Center

EME 1.100, Building 133

Austin, TX 78758-4497

(512) $471-4496$ 
COOLING FOR THE ARAATURB IINDINGS IN A REPETITIVRLY FIRRD AIR-CORE COMPULSATOR

Hsing-Pang Liu

Center for Electromechanics

The University of Texas at Austin Austin, TX 78758-4497

Abstract: The alr-core compulsator was chosen as the pulsed-power supp1y for the CEM-UT Task C (fabrication and demonstration of a stand alone, repetitive fire, compulsator driven, 9-MJ gun system) effort to provide a high current pulse to drive the electromagnetic gun. The 9-MJ projectiles will be fired every $20 \mathrm{~s}$ for a 3 min period of time. During operation, the armature windings will experience a temperature rise of $18.3^{\circ} \mathrm{C}$ per discharge. Since the armature windings must be vacuum impregnated with epoxy for mechanical strength, and the resins generally used for this purpose should not be exposed to temperature above $100^{\circ} \mathrm{C}$, active cooling will be required. Due to the poor thermal conductivity of epoxy ( $50 \%$ of the armature windings), it is very difficult to dissipate the heat generated in the armature conductors.

An analytical model has been built to predict the cooling effectiveness using helium gas flowing through smal1 passages within the windings. Large temperature gradients produced in the windings cannot be tolerated due to the stress generated by the thermal contraction of epoxy; hence, a $245 \mathrm{~K}$ gas temperature was chosen at the armature inlet. In the analysis, the gas temperature, pressure, and velocity distributions along the coolant tube were predicted and the thermal diffusion inside the armature windings was then calculated. The cooling goal is to stabilize the hottest spot temperature of the armature at a given level, which is below the degradation temperature of epoxy, after nine shots.

\section{Introduction}

Design of armature windings for a rotating machine is constralned by electrical performance, the ability to mechanically restrain and attach the windings to the rotor, and thermal considerations. The windings must consist of small diameter metallic wires which are transposed, and will experience a given temperature rise per discharge. The Task $C$ compulsator armature of the Center for Electromechanics at The University of Texas at Austin (CEM-UT) is composed of aluminum wires and epoxy. The epoxy resins will begin to degrade at temperature above $100^{\circ} \mathrm{C}$, which will limit the number of railgun shots. The temperature rise can be reduced at the expense of more armature mass which will introduce more centrifugal load to the armature banding. An active cooling for the armature windings is motivated by the desire to reduce the armature mass and make the windings insensitive to the number of discharges.

Some previous work has been done on the rotor cooling. Armor and Gibney [1] discussed rotor cooling for four-pole generators and showed that their ventilation arrangements would permit considerable increases in unit ratings. They demonstrated that radial-flow gas cooled rotors were simple and efficlent. Abolins and Rieger [2] provided test results of four-pole generators with water cooled stator and rotor windings. In their generators, the windings, connections and terminal bushings were cooled with water, but the heating due to windage, stator iron and stray losses were absorbed by hydrogen gas. Nakayama and Fuzioka [3] investigated flow and heat transfer in the coolant circuit of a water cooled rotor in detail, and tried to predict the pressure drop and heat transfer rate in revolving coolant tubes. Their paper presented some formulas applicable to the design of large water cooled generators. The design of the gas cooled rotors has been revlewed by Gott, et. al. [4], who attributed some of the advances which contributed to the reliability and efficiency of generator rotors to the coolling.

In the CEM-UT Task C compulsator design, forced convection cooling with elther a gas or a liquid can be used to perform the required cooling. However, because the armature rotates with the rotor, the use of liquids can negatively affect the rotor balance if the flow is not distributed evenly. Gases, on the other hand, are of low enough density not to affect the balance. The cooling system being considered utilizes pressurized, chilled hellum gas flowing through small aluminum alloy tubes which pass between the winding conductors.

Cooling tubes make up several 1ayers of the windings and pass from one side of each end turn to the other. Voids between wires and tubes are filled with epoxy by vacuum impregnation. The drawback involved in this cooling arrangement is the low thermal conductivity of the epoxy/aluminum composite (50\% packing factor), and it provides for poor heat transfer by the conductor wires. The heat dissipation rate can be increased by enhancing the thermal conductivity of epoxy. Other than the resin and curing agent, fillers are probably the most common ingredient in epoxy resin systems. Beryllium oxides provide thermal conductivities approximately equivalent to those obtained with the metallic powders; however, the dielectric properties of the system incorporating this filler are not adversely affected. Since the fillers themselves do not shrink, they will also reduce the coefficient of thermal expansion and greatly increase the thermal stability of the system. Even with the improvement in thermal conductivity achieved by adding these fillers, the conductivity of the epoxy matrix is still much less than that of the metal itself; however, it does help the heat conduction appreciably. Unfortunately, this concept was experimentally determined to be infeasible because the resulting highly viscous mixture prevented vacuum impregnation. In order to utilize the vacuum impregnation fabrication technique, high coolant pressure and high coolant flow rate will be required to achieve the cooling.

The original goal of the cooling system was to cool the hottest spot of the windings to ambient temperature within $20 \mathrm{~s}$ after a shot. However, due to the poor thermal conductivity of the epoxy, some preliminary cooling analyses indicated this goal could not be achieved without using a very low temperature coolant and excessive flow rate. In the present design, the armature temperature is allowed to increase a given amount of temperature in the first four or five shots and stabilize at a level which is below the degradation temperature of epoxy. The following section describes the development of the thermal model used to predict the thermal behaviors of the coolant and the armature. 
Thermal Analysis for the Armature Windings

The armature windings can be considered as a combination of many small sections, and each section has a coolant tube at the center. Only a section of the armature is analyzed because of the symmetry. The cooling gas is treated as a one dimensional compressible flow with combined friction and heat transfer. The transient gas temperature, pressure, and velocity distributions down the tube can be predicted with a given initial wall temperature [5]. These boundary conditions can then be used to perform a 3-dimensional thermal diffusion analysis in the armature. For simplicity, some assumptions were made in the analysis:

1. The armature is assumed to be stationary. The pressure rise of the gas due to centrifugal force and extra pressure drop and heat transfer enhancement caused by the tube rotation are neglected.

2. Ideal cooling gas with constant specific heat.

Considering an infinitesimal length of a round tube $d x$, as shown in figure' 1, the energy balance equation for the gas is

$$
\rho\left(\frac{\pi}{4} D^{2}\right) V C_{p} d T_{0}=h(\pi D) d x\left(T_{w}-T_{0}\right)
$$

where

$h=$ convection heat transfer coefficient

$\mathrm{T}_{\mathrm{o}}=$ stagnation temperature

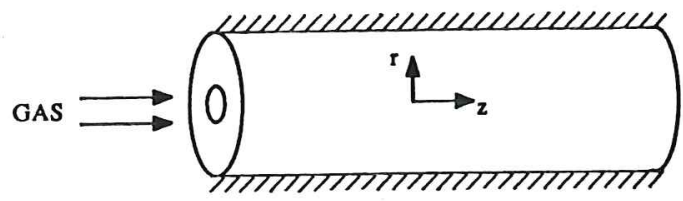

Figure 1. Schematic diagram of a cylindrical annulus

Reynolds analogy relates the heat transfer rate to the frictional loss in a fully-developed turbulent gas f low as

$$
\mathrm{h}=\frac{1}{8} \mathrm{f} \rho \mathrm{VC}_{\mathrm{p}}
$$

In equation (2), a factor of $1 / 2$ was used in reference 5 due to a difference in the definition of friction factor. Substituting this relationship into equation (1), we can obtain

$$
\frac{d T_{0}}{T_{w}-T_{0}}=\frac{f}{2}\left(\frac{d x}{D}\right)
$$

To calculate the pressure, density, temperature, and velocity of a compressible flow, the continuity, momentum, energy equations, and equation of state must be solved simultaneously. Referring to equation (8.40) in [5], the governing differential equation for a compressible gas flowing through a constant area straight tube with combined friction and heat transfer $1 \mathrm{~s}$

$$
\begin{gathered}
d M^{2}=\frac{M^{2}\left(1+k M^{2}\right)\left(1+\frac{k-1}{2} M^{2}\right)}{1-M^{2}}\left(\frac{d T_{0}}{T_{0}}\right)+ \\
\frac{k M^{4}\left(1+\frac{k-1}{2} M^{2}\right)}{1-M^{2}}\left(f \frac{d x}{D}\right)
\end{gathered}
$$

where

$M=$ Mach number

$k=$ the ratio of specific heats

Substituting equation (3) Into equation (4), we get

$$
\begin{gathered}
d M^{2}=\left[\frac{M^{2}\left(1+k M^{2}\right)\left(1+\frac{k-1}{2} M^{2}\right)}{1-M^{2}}+\left(\frac{2 T_{0}}{T_{w}-T_{0}}\right)\right. \\
\left.\frac{k M^{4}\left(1+\frac{k-1}{2} M^{2}\right)}{1-M^{2}}\right] \frac{\mathrm{TT}_{0}}{T_{0}}
\end{gathered}
$$

By assuming a constant wall temperature for a finite length of $x$, we can integrate equation (3) to obtain

$$
\frac{f}{2}\left(\frac{x_{2}-x_{1}}{D}\right)=\ln \left(\frac{T_{w}-T_{o 1}}{T_{w}-T_{o 2}}\right)
$$

Once the Mach number distribution along the coolant tube is solved from equations (5) and (6), the temperature, pressure, and velocity distributions can then be calculated as follows:

$$
\begin{aligned}
& \frac{\mathrm{T}_{2}}{\mathrm{~T}_{1}}=\frac{\mathrm{T}_{02}}{\mathrm{~T}_{01}}\left(\frac{1+\frac{\mathrm{k}-1_{1}}{2} \mathrm{M}_{1}}{1+\frac{\mathrm{k}-\mathrm{M}_{2}}{2} \mathrm{M}_{2}}\right) \\
& \frac{\mathrm{P}_{2}}{\mathrm{P}_{1}}=\frac{\mathrm{M}_{1}}{\mathrm{M}_{2}} \sqrt{\frac{\mathrm{T}_{2}}{\mathrm{~T}_{1}}} \\
& \frac{\mathrm{V}_{2}}{\mathrm{~V}_{1}}=\frac{\mathrm{M}_{2}}{\mathrm{M}_{1}} \sqrt{\frac{\mathrm{T}_{2}}{\mathrm{~T}_{1}}}
\end{aligned}
$$

Equation (7) can be inferred from equations (8.42), $(8.43)$, and $(8.45)$ in [5]. In the above analysis, the friction factor $f$ needs to be estimated first. The tube is divided into a given number of elements. Using a guessed value of $f$ in equation (6), those distributions can be calculated from the previously mentioned equations. Then a new friction factor will be determined, by the average Reynolds number of those fluid sections along the tube, as [6]

$$
\frac{1}{\sqrt{f}}=-2.0 \log \left(\frac{\frac{\varepsilon}{D}}{3.7}+\frac{2.51}{\operatorname{Re} \sqrt{f}}\right)
$$

Iteration is continued until the difference between the calculated and guessed friction factor is less than a set criterion.

In order to have a feel of the quantitative distributions of Mach number, temperature, and pressure of the gas along the coolant tube, a simplified 2-D transient temperature analysis for an armature section was performed. The section was assumed to have a cylindrical annulus geometry, shown in figure 1, with the angular dependence neglected due to symmetry. Through a series of analyses, a $245 \mathrm{~K}$ gas temperature was chosen at the armature inlet so that the cooling requirement can be satisfied and the temperature gradient in the armature can be tolerated. Metal coolant tubes will be required to prevent the epoxy from cracking at the low temperature and high pressure of the cooling gas. Including a stainless stee 1 tube $(0.062 \mathrm{in}$. ID and $0.094 \mathrm{in}$. OD), a cylindrical annulus with $0.364 \mathrm{in}$. OD was used in the following analysis. The armature section was treated as having homogeneous material, and its overall physical and thermal properties were welghted by the volume and thickness of each constituent as follows: 


$$
\begin{aligned}
& \left(\rho C_{p}\right)_{\text {average }}=\frac{\sum_{\substack{1=1 \\
N}}^{N}\left(\rho \mathrm{c}_{\mathrm{p}} \mathrm{v}_{1}\right.}{\substack{\mathrm{N}=1 \\
1}}
\end{aligned}
$$

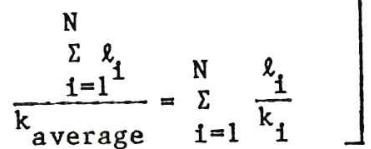

where

$$
\begin{aligned}
N= & \text { the total number of constituents } \\
& \text { In the armature section }
\end{aligned}
$$

It was found that the overall thermal conductivity for the combination of stainless steel, epoxy, and aluminum wire was about $0.49 \mathrm{~W} /(\mathrm{m} \cdot \mathrm{k})$. Apparent $1 \mathrm{y}$, the poor thermal conductivity of the epoxy dominates the radial heat transfer. The governing differential equation, inftial condition, and boundary conditions are summarized as follows:

$$
\frac{\partial^{2} T}{\partial r^{2}}+\frac{1}{r} \frac{\partial T}{\partial r}+\frac{\partial^{2} T}{\partial z^{2}}=\frac{1}{\alpha} \frac{\partial T}{\partial t}
$$

IC

$$
T(z, r, t=0)=T_{0}
$$

BC

$$
\begin{aligned}
& \left.-k A \frac{\partial T}{\partial r}\right)_{r=r_{1}}=h A\left[T_{w}(z)-T_{f}(z)\right] \\
& \left.\frac{\partial T}{\partial r}\right)_{r=r_{0}}=0 \\
& \left.\frac{\partial T}{\partial z}\right)_{z=0}=0 \\
& \left.\frac{\partial T}{\partial z}\right)_{z=L}=0
\end{aligned}
$$

where

$$
\alpha=\text { overa11 average thermal diffusivity }
$$

A finite difference computer code, using alternatingdirection-implicit numerical method, was developed to solve this problem. In the following example, the gas velocity and pressure at the tube inlet were assumed to be $20 \mathrm{~m} / \mathrm{s}$ and $1,500 \mathrm{psia}$, and the tube length and wall roughness were assigned to be $107 \mathrm{in}$. and $64 \mu \mathrm{in}$. respectively. For an initial wall temperature of $323.3 \mathrm{~K}$, which is the estimated temperature of the armature windings after the first rallgun shot, the distributions of velocity, temperature, and pressure are shown in figures 2, 3, and 4. The 2-D transient results indicate that the temperature of the hottest spot in the armature windings only dropped $3.2 \mathrm{~K}$ within $20 \mathrm{~s}$. It 18 understandable that this sma11 temperature drop is caused by the high gas temperature at the tube end and low radial thermal diffusion. However, this result is conservative because the temperature of the epoxy will not increase when the current is introduced. Also, the temperature rise of the coolant tube due to the eddy current loss is not as high as that of the aluminum wires.

After the preliminary investigation, a thorough 3-D thermal model was developed to analyze the transient temperature distribution in the armature windings. In this analysis, the epoxy remained at the same temperature as the wires being heated up by the eddy current 1oss. The heat capacity of the epoxy was then considered, in the later thermal diffusion pro-

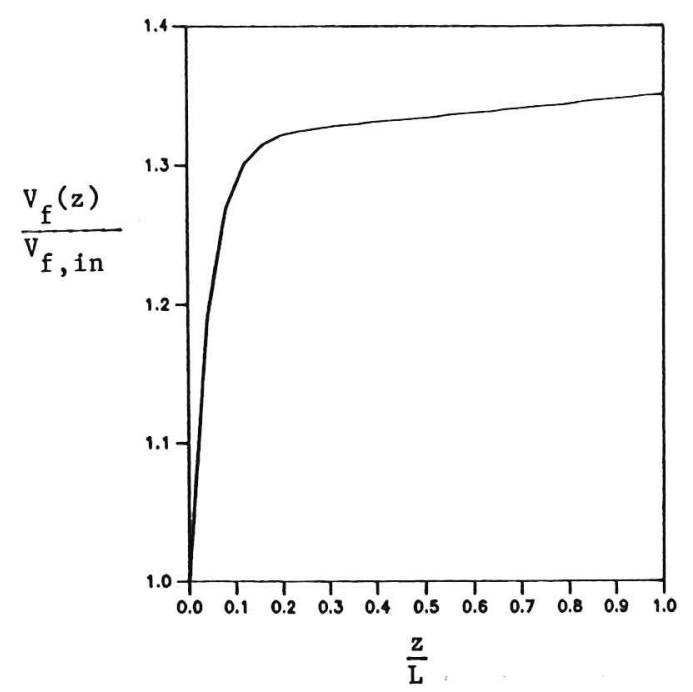

F1gure 2. Velocity vs. distance along the tube

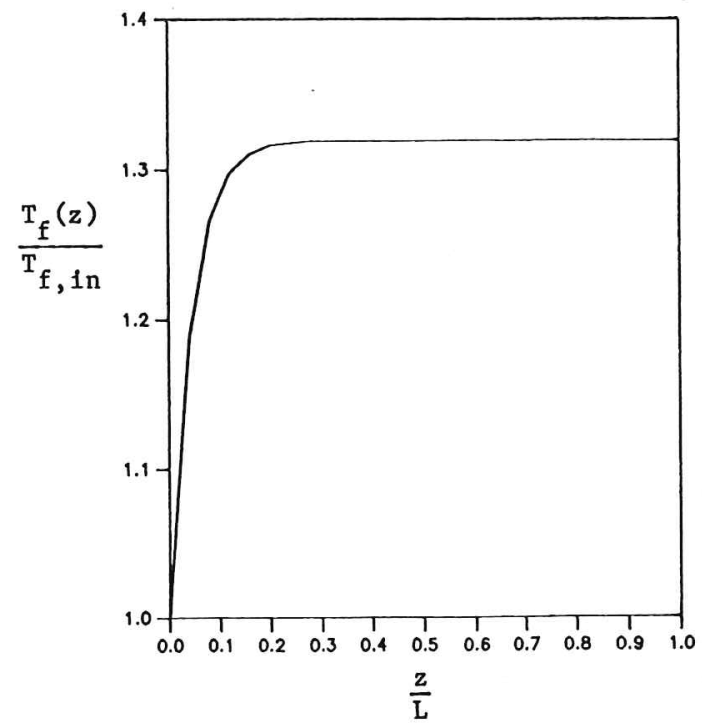

Figure 3. Temperature vs. distance along the tube

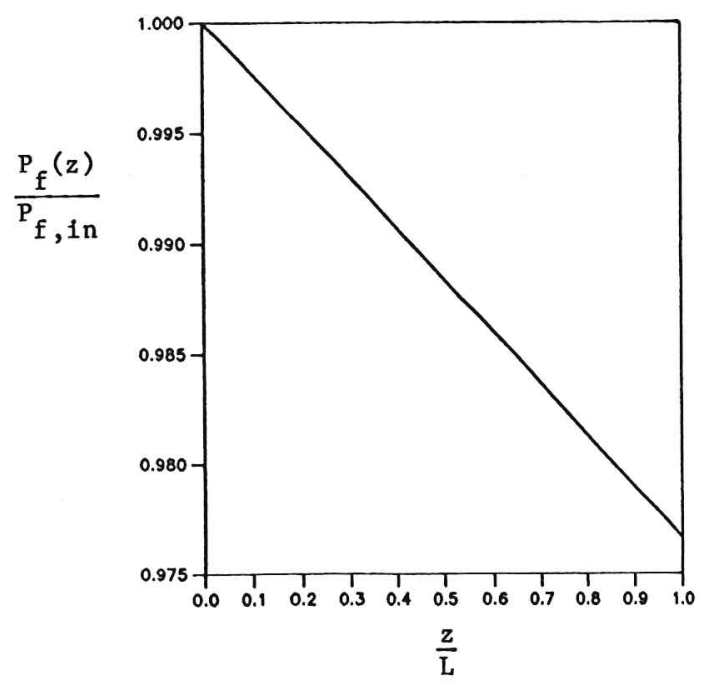

Figure 4. Pressure vs. distance along the tube 
cess, to absorb heat from the wires. Including the end turns and the active length of the armature, the length of the coolant tube was estimated as 107 in. As shown in figure 5 , an armature cross section is divided into six $0.115 \mathrm{in}$. thick conductor layers and three 0.09 in. thick cooling layers. Each cooling layer is responsible for the cooling of its adjacent conductor layers. By assuming a 53\% packing factor (excluding the cooling layers) for the armature, 3.364 in. ${ }^{2}$ conductor area is avallable for each 9.2 in. wide and $1.014 \mathrm{in}$. thick armature. The average density of the cooling layer is designed to match that of the armature; therefore, the spacing between two adjacent coolant tubes is filled up with an appropriate amount of epoxy and finned-type aluminum. The arrangement and dimensions for a square coolant tube with assoclated spacer is indicated in figure 6. High strength aluminum alloy is chosen as the tube material to withstand high gas pressure, and the tube wall thickness is $0.015 \mathrm{in}$.
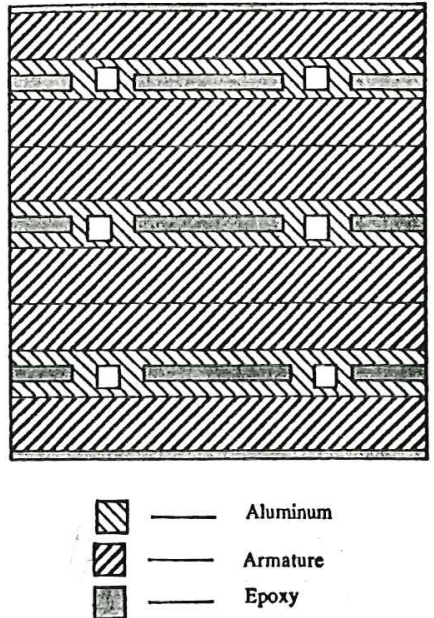

Figure 5. Part of an armature cross section.
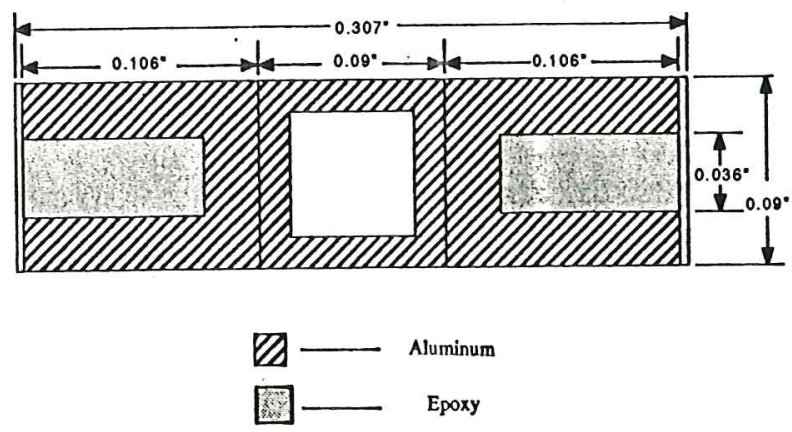

Note:

1. The inside dimensions of the square tube are $0.06 " \times 0.06 "$.

2. The square tube has an uniform wall thickness of 0.015 ".

3. The U-shaped aluminum spacers have a constant width of 0.027 ".

Figure 6. A square coolant tube with associated spacer

Due to the eddy current 1oss, a $18.3{ }^{\circ} \mathrm{C}$ temperature rise of aluminum wires per shot was calculated, and a $3^{\circ} \mathrm{C}$ temperature rise per shot was assumed for the coolant tubes and aluminum spacers. There w111 be 30 tubes for each cooling layer, and a total of 1080 tubes in the cooled armature. A flow rate of $0.9 \mathrm{~kg} / \mathrm{s}$, which corresponded to $17.5 \mathrm{~m} / \mathrm{s}$ inlet velocity, was adapted in the thermal analysis, and the gas temperature and pressure at the inlet of these 107 in. long tubes were $245 \mathrm{~K}$ and 1,500 psia respectively.
In the 3-D heat transfer model, only a quarter of the coolant tube and assoclated spacer and armature were analyzed due to symmetry. As shown in figure 7, the armature was assumed being composed of three alternatively distributed aluminum and epoxy layers. An initial temperature of $305 \mathrm{~K}$ was assumed before the first shot. The temperature rises of aluminum during the subsequent shots were determined by the localized $I^{2} R t$ and specific heat at the corresponding temperature. The computer software package ABAQUS was used to perform the 3-D thermal diffusion, and all the boundary conditions were provided through a user subroutine. For the results presented in the following, the cooling system was assumed to be initiated at the time the first shot was fired.
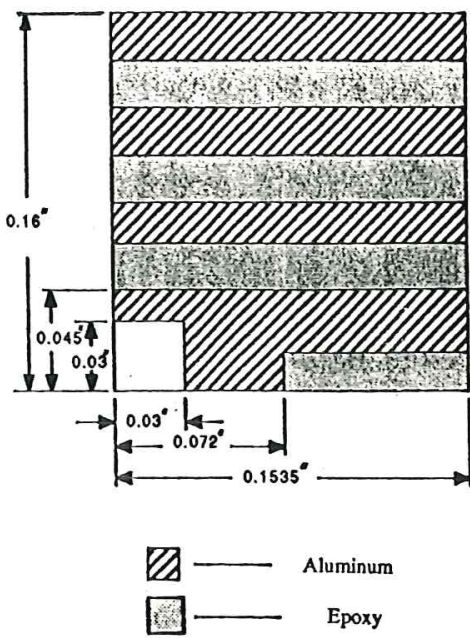

Figure 7. A quarter of a coolant tube, associated spacer and armature used in the 3-D thermal analysis

The temperature histories of the hottest spot, coolest spot, and gas at the exit of coolant tube are shown in figures 8,9 , and 10 respectively. The hottest spot is located at the outer boundary of the passage end, and the coolest spot is located at the interface between the cold gas and tube wall at coolant entrance. During the inftial two cycles, the gas picks up a substantial amount of heat in the passage before reaching the tube exit, which allows no cooling effect near the end of the tube. The temperature drop at the hottest spot in the beginning two cycles, indicated in figure 8 , results from the heat diffusion into the adjacent epoxy layer. By examining figure 10 , it can be found that the gas temperature at the tube exit becomes lower and lower after two or three shots, and then the cooling effect can be felt at the very end of the tube. The hottest spot temperature reaches the peak temperature $347 \mathrm{~K}$ after the fourth shot, and then declines as the profile shown in figure 8. Comparing figure 8 with figure 9, a maximum temperature difference of $100^{\circ} \mathrm{C}$ exists in the $107 \mathrm{in}$. long armature. In the analysis, the gas enters the tube at a velocity of $17.5 \mathrm{~m} / \mathrm{s}$, and exits the tube at about $23 \mathrm{~m} / \mathrm{s}$ due to the heating acceleration. Without considering the rotational effect, a 25 psi pressure drop in the tube is calculated.

This cooling analysis was originally developed to treat the compressible gas flow in the coolant tube. As mentioned earlier, the intended goal at the beginning of this cooling system design was to cool the hottest spot of the windings to ambient temperature within $20 \mathrm{~s}$ after a shot, and nitrogen gas with $80 \mathrm{~K}$ inlet temperature was chosen at that time. With excessive flow rate required, the cooling analy- 
sis indicated that the flow choking was a problem. Also, before the coolant reaches the inlet of the armature windings, a significant temperature rise of the nitrogen gas due to radial compression in the coolant delfvery line made the cholce of low temperature nitrogen gas infeasible. To avold these problems, hellum gas with lower flow rate was used in the design.

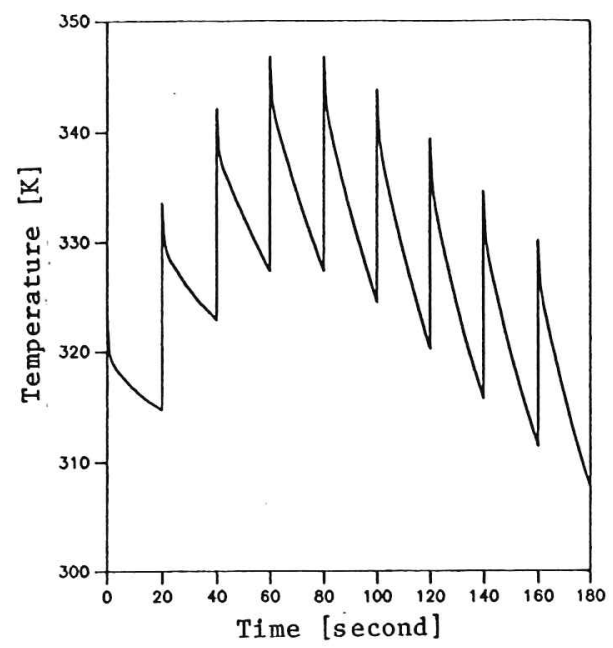

Figure 8. Temperature history of the hottest spot of the armature

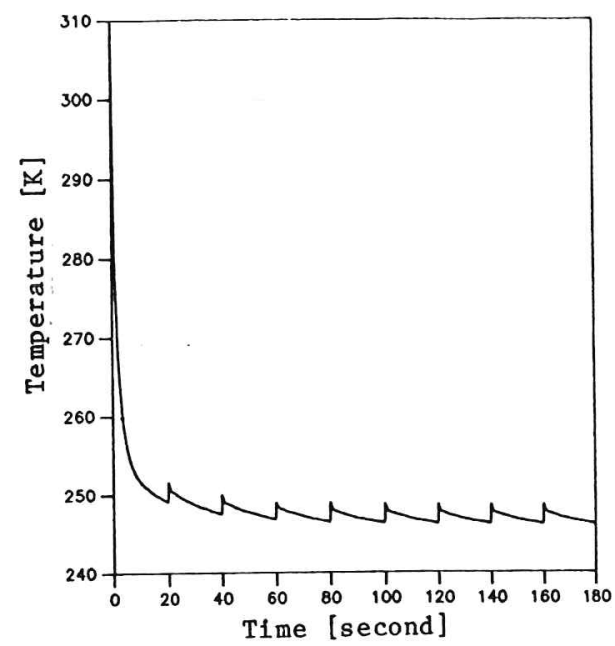

F1gure 9. Temperature history of the coolest spot of the armature

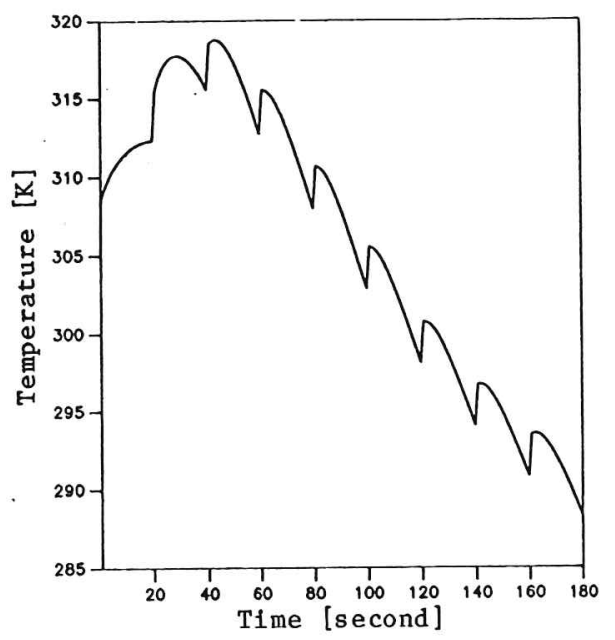

Figure 10. Temperature history of the gas at the tube exit
In the 2-D and 3-D transient analyses for an armature section, the armature temperature and coolant inlet conditions are similar, and the averaged Reynolds number, Mach number, and Prandt1 number of the hellum gas along the coolant tube are 30,500 , 0.022 , and 0.67 , respectively. For the low Mach number flow in the cases investigated, the elaborate cooling analysis presented in this paper may not produce results much more accurate than those of a simplified incompressible flow analysis, but this analysis will particularly be useful for designs that require high flow rate.

The provision of a constant gas flow rate and gas temperature at the tube inlet is very difficult due to the variations of rotor speed and gas temperature at the exit of the tube. Also, for the pre-armature and post-armature sections in the coolant delivery line, the pressure, temperature, and velocity of the gas w111 experience changes because of the compression, expansion, and heat exchange with the coolant passage. These 1ssues will be addressed in the following section.

\section{Description and Design of Coolant Delivery System}

The basic 1dea of this cooling system is that the cold hellum gas is delivered through the Inner pipe of a concentric annular shaft through one end closure. The gas is then radially compressed (self-compression due to rotor rotation) as it flows to the armature inlet manifold, forced through the armature to the outlet manifold, and radially expanded to the annular space of the shaft. The whole closed loop is completed by two rotating seal couplings, an external heat exchanger and an external compressor.

In the armature coolant delivery system (inside the compulsator rotor), radial centrifugal compression of the cooling gas increases both its pressure and temperature, while the radial expansion section decreases these parameters. The net difference between these two processes will determine the shaft work required to drive this cooling system. The efficiencles of these compression and expansion processes play critical roles in the overall delivery system design.

First, an estimation of pressure and temperature rises due to compression is made. The centrifugal passages 1mpart kinetic energy to the gas by increasing the gas peripheral velocity through rotation. This kinetic energy results in a static pressure rise and a temperature rise in the cooling gas. The kinetic energy is a function of the square of the velocity; therefore, the pressure produced by such a radial passage is directly proportional to the square of the mechanical tip speed of the passage. The gas compression can be treated as a polytroplc process which is a variable entropy process with heat transfer taking place. The polytropic process can be expressed as

$$
\mathrm{PV}^{\mathrm{n}}=\text { constant }
$$

The value of " $n$ " is a function of the constant "k" (ratio of specific heats $\mathrm{C}_{\mathrm{p}} / \mathrm{C}_{\mathrm{v}}$ ) for the gas and the polytropic effictency, $n_{p}$. The relationship can be expressed by the following equation:

$$
\frac{\mathrm{n}}{\mathrm{n}-1}=\left(\frac{\mathrm{k}}{\mathrm{k}-1}\right) n_{\mathrm{p}}
$$

The gas behavior in the centrifugal passages can be described by the equation of motion of a compressible Newtonian fluid, with constant thermal properties. In cylindrical coordinates, the equation of motion is 


$$
\begin{gathered}
\frac{\partial v_{r}}{\partial t}+(v \cdot \nabla) v_{r}-\frac{1}{r} v_{\theta}^{2}=-\frac{1}{\rho} \frac{\partial p}{\partial r}+g_{r}+v \\
\left(\nabla^{2} v_{r}-\frac{v_{r}}{r^{2}}-\frac{2}{r^{2}} \frac{\partial v_{\theta}}{\partial \theta}\right)
\end{gathered}
$$

and the appropriate equation of state is

$$
\mathrm{PV}=\mathrm{ZmRT}
$$

where

$$
\mathrm{Z}=\text { compressibility factor }
$$

In the following derivation, the gas is assumed to follow the passage's contour smoothly; in other words, no "slip" occurs at the exit of the radial passage. After eliminating negligible terms and assuming that the radial velocity component is small in comparison with the tangential component in equation (13), then combining with equation (14) and integrating along the radial direction, the following result is obtained:

$$
\frac{1}{2} U_{2}^{2}=\operatorname{ZRT}_{1}\left(\frac{n}{n-1}\right)\left[\begin{array}{c}
\frac{n-1}{n} \\
\left(\frac{p_{2}}{p_{1}}\right)-1
\end{array}\right]
$$

where

$$
\begin{aligned}
\mathrm{U}_{2}= & \text { mechanical tip velocity at } \\
& \text { a rmature mean radius, }(550 \mathrm{~m} / \mathrm{s})
\end{aligned}
$$

The temperature ratio and pressure ratio for a polytropic process can be related as follows:

$$
\frac{T_{2}}{T_{1}}=\left(\frac{p_{2}}{p_{1}}\right)^{\frac{n-1}{n}}
$$

Once the coolant goes through the armature windings, it must overcome the centrifugal force in order to flow back to the central exhaust pipe. This can be treated as a radial expansion process. The previously mentioned equations used for compression are still valid in this case except the determination of " $n$ ", which must be defined as follows:

$$
\frac{\mathrm{n}-1}{\mathrm{n}}=\left(\frac{\mathrm{k}-1}{\mathrm{k}}\right) n_{\mathrm{p}}
$$

Due to the temperature rise of the gas during heat addition from the armature, the pressure rise due to compression is greater than the pressure drop due to expansion. Also, the net difference between them becomes larger if the pressure level in the delivery system is high and, as a result, this pressure gain may be large enough to overcome the rest of the system pressure drop. If this is the case, the whole delivery system is self-pumping, and an external compressor will not be necessary. The pressure level of the gas is very 1mportant; the higher the pressure is, the larger the pressure gain will be. For a fixed flow rate, a high pressure also means a low velocity and consequent1y a low pressure drop. Currently a pressure level of $1,150 \mathrm{psia}$ and a velocity of $26 \mathrm{~m} / \mathrm{s}$ at the inlet of rotor is planned and used in the calculations.

The heat exchanger is designed to have hellum gas flowing through pipes which are immersed in a pressurized liquid nitrogen storage tank. Heat is removed by the vaporization of liquid nitrogen. To increase the efficlency of the heat exchanger, the boll off from liquid nitrogen can be used to precool the hot incoming hellum gas before it enters the liquid nitrogen tank.

Rotation generally improves heat transfer, and the increase is greatest in the developing flow region and for lower flow rate[7]. For a coolant passage rotating about a parallel axis, the dominant effect is on inlet swirl induced by rotation, combined with Corfolis acceleration which is important in the entrance region as a source for the creation of secondary flows [8]. The overall pressure loss in the rotating passage wi11 also Increase with the increased flow resistance due to the Coriolis force and centrifugal buoyancy (caused by density variation of fluid in a centrifugal field). The increase of flow resistance due to rotation is governed by the Rossby number which is the ratio of fluid inertial momentum to Coriolis acceleration. Empirical equations [3] provide the relationships between the flow resistance coefficient and Rossby number. But it is belleved that the rotational effect is insignificant in the CEM-UT system's small gas passages because the development of secondary flow is restricted in the small coolant tubes.

The radial compression and radial expansion processes in the coolant delivery system control most of the pressure and temperature changes of the gas coolant. Design of these radial coolant passages is therefore crucial in the overall armature windings cooling system. Stability of the gas coolant is another major concern in the delivery system design. In general, "surge" is the major phenomenon which causes unstable operation of centrifugal compression [9]. Surge is a condition in which the pressure developed by the compression process is less than that in the system downstream. The volume flow rate into the compression passage is thereby reduced to the point that the velocity of the gas going through the radial compression passage also decreases. When the velocity becomes too low, the compression process can no longer perform stably, and a flow reversal can result. Surge is a cyclic phenomenon; as the gas flows back into the compression passage, the pressure losses within the passage $f a 11$ and the discharge pressure of the gas rises, causing the forward flow of gas to be resumed. This back-and-forth flow can cause catastrophic damage to the coolant delivery system. Maintaining a minimum flow through a centrifugal compressor is necessary to prevent surge, and antisurge control systems must be implemented. Patlovany and Focke [10] described the installation of a control system on a large alr compressor, which was used to protect the compressor from surge.

Another potential problem associated with the coolant delivery system is the inlet and outlet manifolding within the armature windings. It may be difficult to attain an even distribution of flow in all the coolant passages. Achieving flow equalization in the passages involves a study of manifold configuration, passage size, and spacing. A thorough 1iterature survey indicated that Bajura [11] has analyzed the flow distribution in manifolds. H1s mathematical model describing the flow behavior at a discrete branch point was formulated in terms of momentum balance along the manifold. The uneven flow distribution was predicted and favorably compared with the experimental results. Researchers of Stanford University [12] analyzed the oblique flow headers for heat exchangers and provided a series of closed form solutions needed to specify the inlet and exit header shapes which yield a unfform flow distribution. Experimental results supported the predictions quite 
well ( $\pm 5 \%$ nonuniformity). Their analysis was based on a quasi two-dimensional approach and the solution was restricted to frictionless flow and constant-width outlet headers only. In the CEM-UT system, restrictions may be required in the manifolds to regulate the pressure difference across each passage, or the supply and exhaust manffolds may be tallored to malntain a constant pressure difference across windings.

\section{Conclusions}

A thermal mode1 has been built to theoretically analyze the cooling in the armature windings of a repetitively fired alr-core compulsator. Helium gas is chosen as the coolant, and the temperature, pressure, and velocity distributions of the gas along the coolant passage are predicted, which are used to solve for the thermal diffusion in the armature. Radial heat diffusion in the combination of aluminum wires, epoxy, and tube dominates the heat transfer mechanisms, and determines whether the cooling goal can be achleved; therefore, the radial dimension is very critical and it is important to minimize the armature thickness.

The design of the coolant delivery system is currently in progress. Those potential problems involved in the delivery design are addressed, and approaches to overcome the problems are discussed. The critical points in this design are the coolant sectors for radial compression and radial expansion, which control most of the pressure/temperature rise and drop. For an optimal coolant delivery system, both the power required to drive the cooling system and the external heat exchanger capacity should be minimized.

\section{Nomenclature}

$A=$ cross-sectional area, $m^{2}$

$\mathrm{C}_{\mathrm{p}}=$ constant-pressure spec1fic heat, $\mathrm{J} /(\mathrm{kg} \cdot \mathrm{K})$

$\mathrm{C}_{\mathrm{v}}=$ constant-volume specific heat, $\mathrm{J} /(\mathrm{kg} \cdot \mathrm{K})$

$\mathrm{D}=$ diameter, $\mathrm{m}$

$f=$ friction factor

$\mathrm{g}=$ acceleration of gravity, $\mathrm{m} / \mathrm{s}^{2}$

$\mathrm{h}=$ convection heat transfer coefficient, $\mathrm{W} /\left(\mathrm{m}^{2} \cdot \mathrm{K}\right)$

$\mathrm{k}=$ specific heat $\mathrm{ratio}, \mathrm{C}_{\mathrm{p}} / \mathrm{C}_{\mathrm{v}}$

$=$ thermal conductivity, $\mathrm{W} /(\mathrm{m} \cdot \mathrm{K})$

1 = thickness, $\mathrm{m}$

$\mathrm{L}=$ axial length, $\mathrm{m}$

$\mathrm{m}=$ mass, $\mathrm{kg}$

$M=$ Mach number

$\mathrm{n}=$ polytropic exponent

$\mathrm{p}=$ pressure, $\mathrm{N} / \mathrm{m}^{2}$

$r=$ radial distance in cylindrical coordinate system, m

$r_{1}=$ inner radius, $m$

$r_{0}=$ outer radius, m

$\mathrm{R}=$ gas constant, $\mathrm{J} /(\mathrm{kg} \cdot \mathrm{K})$

$\operatorname{Re}=\operatorname{Reynolds}$ number

$t=t$ ime, $s$

$\mathrm{T}=$ temperature, $\mathrm{K}$

$\mathrm{U}_{2}=$ peripheral velocity, $\mathrm{m} / \mathrm{s}$

$v_{r}, v_{\theta}=$ cylindrical polar velocity components

$\mathrm{v}=$ velocity, $\mathrm{m} / \mathrm{s}$

$=$ volume, $\mathrm{m}^{3}$

$\mathrm{x}=$ length, $\mathrm{m}$

$z=a$ spatial coordinate in cylindrical coordinate system, m

$\mathrm{Z}=$ compressibility factor

$\rho=$ density, $\mathrm{kg} / \mathrm{m}^{3}$

$\varepsilon=$ wall roughness, m

$\alpha=$ thermal diffusivity, $\mathrm{k} / \mathrm{\rho c}, \mathrm{m}^{2} / \mathrm{s}$

$\nu=$ kinematic viscosity, $\mathrm{m}^{2} / \mathrm{s}$

$\theta=$ angular coordinate in cylindrical coordinate system

$n_{p}=$ polytropic efficiency
Subscripts

0 = stagnation state

= Initial condition

$\mathrm{w}=\mathrm{wal1}$

$\mathbf{f}=\mathrm{f} 1 \mathrm{uld}$

in $=$ inlet

\section{Acknowledgements}

The author wishes to thank A1an Wa11s, Don Bresie, and Bill Brinkman for their useful discussions and technical support. This research is sponsored by the Defense Advanced Research Profects Agency and the U.S. Army Research, Development, and Engineering Center.

\section{References}

[1] Armor, A. F., and Gibney, J. J., III, "Direct Conductor-Cooling of Large Steam Turbine-Generator 4-Pole Rotors," IEEE Transactions on Power Apparatus and Systems, Vol. PAS-93, No. 2, 1974, pp. 477-486.

[2] Abolins, A., and Rleger, F., "Test Results of the World's Largest Four-Pole Generators with Water-Cooled Stator and Rotor Windings," IEEE Transactions on Power Apparatus and Systems, Vol. PAS-94, No. 4, 1975, pp. 1103-1110.

[3] Nakayama, W., and Fuzloka, K., "F1ow and Heat Transfer in the Water-Cooled Rotor Winding of a Turbine Generator," IEEE Transactions on Power Apparatus and Systems, Vo1. PAS-97, No. 1, 1978, pp. 225-231.

[4] Gott, B. E. B., Kaminski, C. A., and Shartrand, A. C., "Experience and Recent Development with Gas Directly Cooled Rotors for Large Steam Turbine Generators," IEEE Transactions on Power Apparatus and Systems, Vo1. PAS-103, No. 10, 1984, pp. 2974-2981.

Shapiro, A. H., The Dynamics and Thermodynamics of Compressible Fluid Flow, Ronald Press, New York, 1958.

[6] White, F. M., Fluld Mechanics, McGraw-H111, New York, 1979.

[7] Stephenson, P. L., "An Experimental Study of Flow and Heat Transfer in a Duct Rotating About a Parallel Axis," XIV Symposium of The International Center for Heat and Mass Transfer, Dubrovnik, Yugoslavia, 1982.

Johnson, A. R., and Morris, W. D., "Pressure Loss Measurements in Circular Ducts Which Rotate About a Parallel Axis," XIV Symposium of The International Center for Heat and Mass Transfer, Dubrovnik, Yugoslavia, 1982.

[9] Boyce, M. P., Gas Turbine Engineering Handbook, Gulf Publishing Company, 1982.

[10] Patlovany, D., and Focke, A. B., "Predictive Surge Control and Optimization for a Centrifugal Compressor," ASME Journal of Turbomachinery, Vo1. 108, July 1986, pp. 82-89. Bajura, R. A., "A Model for Flow Distribution In Manifo1ds," ASME Journal of Engineering for Power, January 1971, pp. 7-12.

London, A. L., Klopfer, G., and Wolf, S., "Oblique Flow Headers for Heat Exchangers," ASME Journal of Englneering for Power, Ju1y 1968, pp. 271-286. 